\title{
Infrared Printing Technique for the Security Marking of Traceability Certificates for Meat Products
}

\author{
Dražen Crčić, Jana Žiljak Gršić, Denis Jurečić
}

\begin{abstract}
The focus of this paper is the introduction of new security elements into the authenticity of information on documents which concern the traceability of products. This article proposes dual marking using the infrared printing technology, which involves differences between the visible and infrared spectrum and separate detection of certain information with an infrared camera. Each meat product is assigned a unique designation, embedded in the document and hidden from the naked eye, in order to prevent changing, scanning or modifying with currently known techniques. A product receives a certificate with a security approval which carries information about the complete traceability of the product. A new central data registry which collects information from all necessary sources is proposed. The web portal and the accompanying database are designed to work in the cloud. The security of the exchange of data between the server and the user is ensured. The information about the traceability and the delineation between the visible and infrared hidden are approved and coordinated by the relevant authorities, breeders, distributors and sellers. The design of the entire information presents completely transparent information about the products to the end consumer.
\end{abstract}

Keywords: central data registry; Certificates of Traceability; INFRAREDESIGN; near infrared spectrum; security marking

\section{INTRODUCTION}

The packaging of a product is loaded with information about the product, quality, method of application and other possible legally required elements. Designers have to find space for a lot of data on the packaging of a certain product.

Conventional security print products involve the use of ultraviolet and infrared colorants and papers with security properties, such as a watermark, embedded threads or finishing with foil printing or relief printing. All these techniques increase the cost of the graphic product and are performed in specially authorized printing offices. The emergence of forgeries and attempts at imitation of the existing security technologies are the sign than these conventional techniques are outdated and that new solutions for security printing are needed. This article proposes the INFRAREDESIGN ${ }^{\circledR}$ technology, which is expanded into individual design though the use of line computer graphics. The IRD CERTIFICATE includes a high level of planning for the hidden information on the packaging. The hidden information is implemented together with other graphic elements in the same printing process for the graphic product. The contribution to security printing is in the design with dual images embedded within each other [1]. This kind of printing technology does not raise the cost of the printing product.

The hidden information is detected with an infrared camera, infrared photo camera or security cameras, such as those that surround us in cities and in stores [2]. These cameras show the hidden image, code or individual marking, none of which can be scanned or photocopied. The system includes the INFRAREDESIGN patent for the individualization of documents during the printing phase and within the information system which creates the packaging.

IRD protects the product and the producer, can be integrated without changing the existing design, i.e. the product registration, and can be applied to all necessary materials. One of the advantages is that the protection is created in the same process as the packaging for the product or the part of product we want to identify.

\section{INFORMATION ON THE TRACEABILITY DOCUMENT}

There are official programs for the digital record of all these processes, usually determined by the relevant ministries. It is very difficult or even impossible to determine the complete traceability of the origin of meat products in one place. The technologies of traceability of meat products vary in the information provided and in the security marking. This is due to the fact that different programs for different stages of rearing are not connected with each other and do not cover all the processes up until the packaging. There is no one document or one procedure for recording data about the traceability of the production and the product. The final label on the product provides only superficial information about the origin of the product.

Unambiguous security marking of meat on its path from the slaughterhouse to the retail stores is proposed. Currently in this segment only general data and LOT numbers are used, which cannot unambiguously determine the detailed origin of a certain product.

The new central data registry (SMP) collects and consolidates data which confirms traceability in one place. The data is exchanged through the internet - through security https protocol and an additional communication key. This ensures the complete safety of data exchange through a public network. The current registry of information is partial. The tracking of animals and their identification numbers is only done by farmers and slaughterhouses. The data is located in the data registries of the Croatian Agricultural Agency (HPA). Tracking is currently not conducted at the time of packaging. Therefore, it is necessary to ensure that SMP collects additional information about the packaging. This information contains the unique packaging number and 
the corresponding animal identification number. The new central registry can collect this data in two ways:

- automatically through program integration with the HPA registry,

- manually by farmers, slaughterhouses and packaging companies, who send all locally processed data to the new central registry.

A separate module has been developed for the manual transfer of data. It is run as a web application through an internet browser. The user receives access data and uploads data. The module performs an automatic conversion of the data structure in order to save them in the SMP.

Each product on retail shelves would get its own universal designation. Products would be marked with QR codes [3]. The universal code has a link to the SMP database. The universal code also contains the information about the company and the date of packaging.

Consequently, all information about the meat product can be accessed through the universal designation and previously collected information. The information is available on a separate SMP web portal. The access to information is protected with security protocols and can be given only to designated persons.

If the products are finished, imported products, the data will not contain all the information. However, if the rearing was partially or entirely conducted in Croatia, the information would contain all other data. Through the product designation, which would be visible on the packaging, the following parameters can be accessed:

\section{Packaging label: 7295650472-1583485950-73913-2 \\ Markets: Company xxx \\ Type of meat: Beef \\ Born: 05/02/2018 Romania \\ Breed: Crossbreeds \\ Life number: RU 234123678 \\ Fattened: 12/03/2018 - 23012020 Croatia (Farm xxx) \\ Date of slaughter: 01/24/2020 Croatia \\ Slaughterhouse mass: $247 \mathrm{~kg}$ \\ Packing Date: 01/24/2020 \\ Use up to: 02/10/2020 (BEEF)}

The unique designation of the product is generated by using four parameters. The first parameter is the designation of the packaging company. The second parameter is the time of packaging according to the "Unix time" (the number of seconds elapsed since 01/01/1970). The third parameter is a random number, and the forth is a control mark. The program code stated below represents the algorithm for generating a unique designation. The code is made for the PHP programming language. It can be used as an addition to the current program solutions in the packaging process or as a separate module. It is the principal link between the product designation and the traceability of the product.

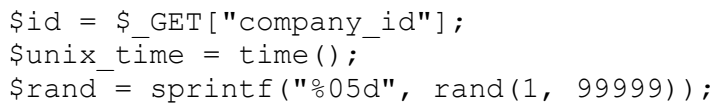

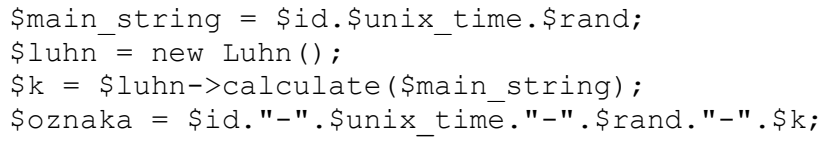

\section{INFRAREDESIGN OF DUAL EMBEDDED GRAPHICS}

The implementation of IRD documentation would bring multiple benefits. End consumers would have detailed information about the origin of the product. Complete reports would enable the authorities to better track and control production and distribution. Origin control would be greatly simplified. Every company that conducts the packaging of products is required to send the new designation and the animal identification number to the central data registry. This makes it possible to determine for every product: where the animal was born, what farm(s) it was reared at and when and where the meat was classified.

With the IRD certificate, each product on store shelves receives its own, innovative, universal sign. Products are marked with dual QR codes with additional IRD protection [3]. A design with two different QR codes is printed in one spot. Within the code for the visible spectrum the QR code is embedded read on the screen of the camera for the infrared light spectrum. The security of information in both codes is achieved. The implementation of (paper) IRD documents would bring multiple benefits. IRD documentation is used in two forms. The first use is for manual input of information collected for the central register, i.e. for the internet database. The second use is for the end user when he or she receives the packaged product.

The line graphics have curved elements at the edges of the letters "TYPE OF MEAT: BEEF". The oblique line covers more space, causing the effect of typography recognition. The certificate is transparent and only $60 \%$ of its surface is covered with color. The colors in the curved lines vary based on a linear congruential generator with a six-digit seed and a modulus of integer values. The second image from the IRD plan, invisible to the naked eye, will be recorded with a camera for the infrared spectrum. The initial image is a continuous photograph. By combining it with curved line graphics, the hidden image will appear only in parts of the image (Figure 4). Positive gray values exist only where there are twin colorants defined in curved line graphics - in other words, only where the replacement of process colorants cyan, magenta and yellow with the carbon black color according to the method of VZ separation (the special part of GCR/Gary Color Reduces process) is possible.

For the purposes of this article, the recipes for twin colors for the carbon black color with $40 \%$ coverage on polypropylene material (1) have been experimentally calculated. In our lab, the experimental plan covers between 80 and 120 recipes for twin colorants. Some printing techniques and their corresponding color tones require many experiments, which include the examination of the dependence of the layer thickness of a specific process color, transparency, the type of the raster form [4] and the common property of light absorption. The results (with the condition of $\Delta E<3$ ) of different recipes for color tones are shown as a regression 
equation of the dependence of $\mathrm{X}_{40}$ on $\mathrm{X}_{0}$ [1]. The reduction of every C, M, Y colorant in $\mathrm{X}_{40}$ shows a mutual dependence on the other two colors from $\mathrm{X}_{0}[1]$.

$$
\begin{aligned}
& \mathrm{C} 40=-0.0222 * \mathrm{Y}-0.175 * \mathrm{M}+1.244 * \mathrm{C}-30.3 \\
& \mathrm{M} 40=-0.055 * \mathrm{Y}+1.105 * \mathrm{M}+0.133 * \mathrm{C}-34.1 \\
& \mathrm{Y} 40=1.124 * \mathrm{Y}-0.0802 * \mathrm{M}+0.0172 * \mathrm{C}-33.2
\end{aligned}
$$

For the first five colors (Fig. 1) of the first five curved lines the values are given from relation (1) in Tab. 1. Model (1) can serve for some other plans, provided that the parameters in the equations are valid for digital printing with toner on a transparent material.

Table 1 Recipe for the first five twins according to model (1):

\begin{tabular}{|c|c|c|c|c|}
\hline & $\mathrm{X} 0: \mathrm{C}, \mathrm{M}, \mathrm{Y}, \mathrm{K}=0$ & $\mathrm{X} 40: \mathrm{C}, \mathrm{M}, \mathrm{Y}, \mathrm{K}=40$ & $\begin{array}{c}\text { Difference } \\
\mathrm{X} 0-\mathrm{X} 40\end{array}$ & \\
\hline 1. & $51,95,79,0$ & $15,73,49,40$ & $36,22,30$ & \\
\hline 2. & $95,54,51,0$ & $76,35,21,40$ & $18,18,30$ & \\
\hline 3. & $61,44,82,0$ & $36,18,56,40$ & $25,26,26$ & \\
\hline 4. & $85,73,90,0$ & $61,53,64,40$ & $24,20,26$ & \\
\hline 5. & $58,62,44,0$ & $30,40,12,40$ & $28,22,32$ & \\
\hline
\end{tabular}

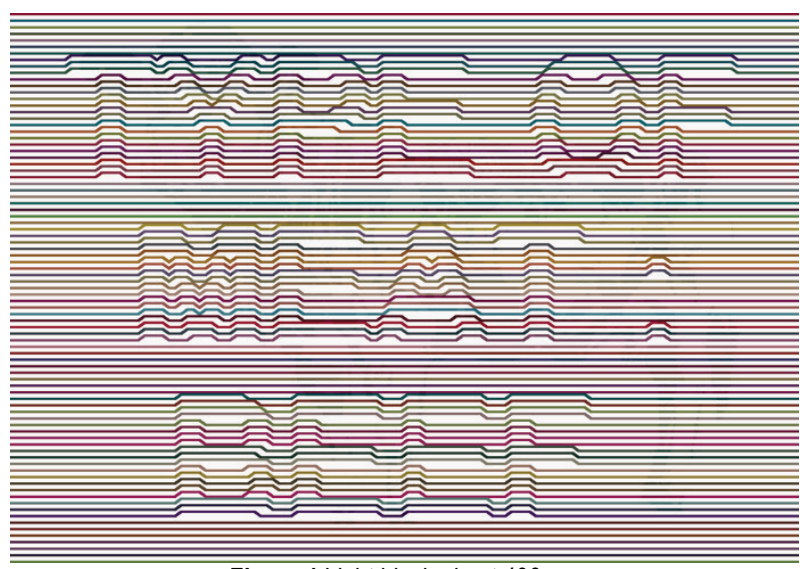

Figure 1 Light blockade at $400 \mathrm{~nm}$

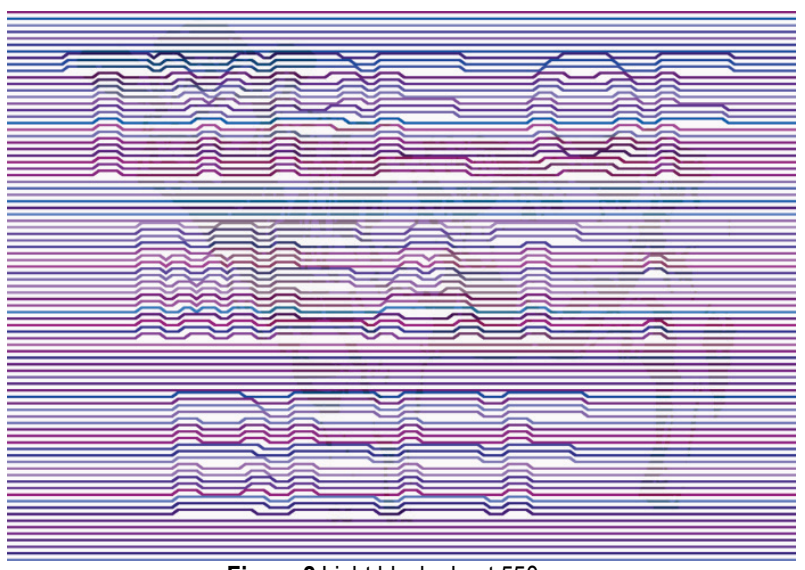

Figure 2 Light blockade at $550 \mathrm{~nm}$

The colors in the lines gradually disappeared through filtering: yellow at $550 \mathrm{~nm}$, magenta at $650 \mathrm{~nm}$ and cyan at $780 \mathrm{~nm}$ [5]. The continuous changes of light filtering are observed and detected with an animation given at: jana.ziljk.hr/beef.mp4 [6], where the images from 24 light blockades from the visible and near-infrared spectrum between 400 and $1000 \mathrm{~nm}$ were combined [7].

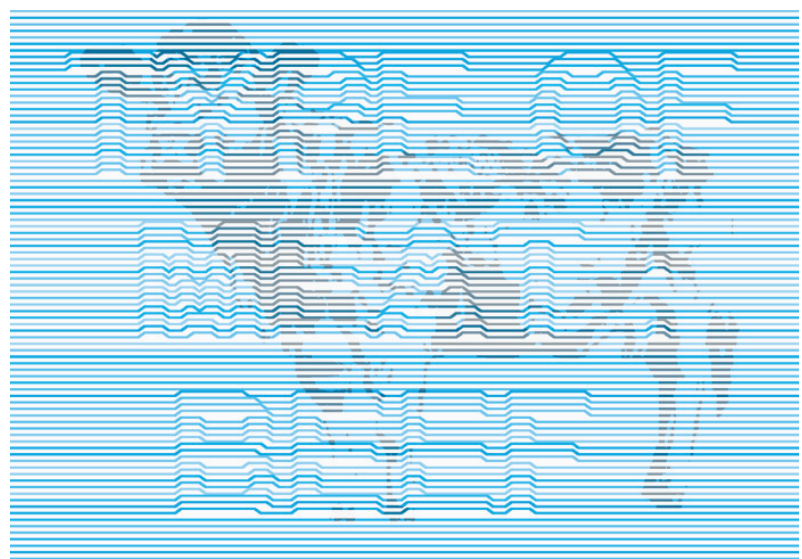

Figure 3 Light blockade at $700 \mathrm{~nm}$

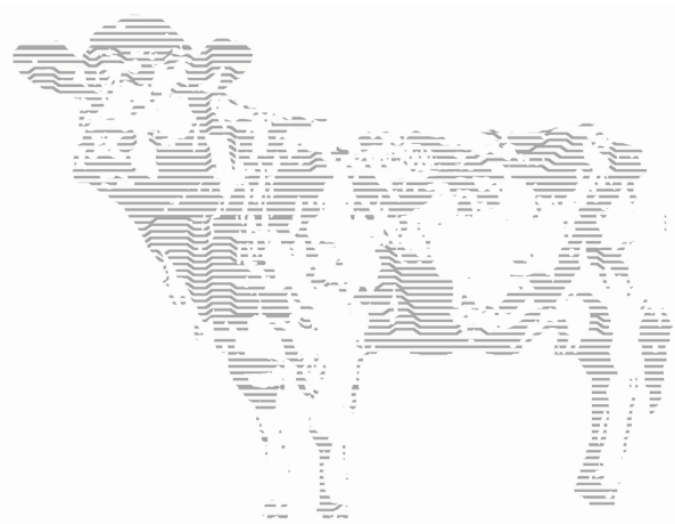

Figure 4 Light blockade at $1000 \mathrm{~nm}$

The security video animation shows the appearance of the hidden image already at $650 \mathrm{~nm}$, but this image can never become the basis for creating a new, falsified dual image. The proposed innovation is the combination of computer graphics as curved lines which select the information of the hidden image. Our eyes see the imprint identical to the blockade at $400 \mathrm{~nm}$ (Fig. 1), just as an RGB photography does. The photograph with the $\mathrm{Z}$ camera is identical to Fig. 4 .

\section{DISCUSSION AND CONCLUSION}

Infrared intrigues with its simultaneous visibility and invisibility and opens up entirely new possibilities unimaginable prior to its invention. This invention arouses interest and offers numerous possibilities for graphic design solutions. IRD motivates us to think about the application in the system of protection against counterfeits.

The hiding of the information in order to protect the packaging is ensured in the infrared and visible spectrum. Authenticity is proved using spectral analysis of colorants on the graphic product. IRD brings innovation in the design of packaging and the protection against copying. Photocopying machines do not recognize the $Z$ spectrum $(1000 \mathrm{~nm})$ in the infrared area or the technology of the separation of two pieces 
of information which are physically in the same place. IRD opens up the possibility of applying hidden barcodes with multi dye twin color for the protection against falsifying. These codes would also be invisible to the naked eye and detectable only with a camera for the infrared spectrum.

The new central data registry (SMP) collects data from all the necessary databases and sources. The SMP is in the cloud. The data is exchanged through the internet - through security https protocol and an additional communication key. This ensures the complete safety of data exchange. JSON structure of data with REST API methods is used.

Infrared certificates serve as receptacles. They are physically organized information which gives the end user an additional sense of security concerning the information about the traceability of products.

Information about the traceability would be provided as needed to relevant authorities, breeders, distributors and sellers. It is also possible to present the whole information to end consumers as completely transparent information about the sold products.

This would increase the visibility of Croatian farmers. There is a problem in certain parts of the industry - the differences between the quality of meat of animals born and reared outside of Croatia and the quality of meat of animals born outside of Croatia and those entirely reared in Croatia are not sufficiently recognized. With transparent information about the traceability, such differences would immediately be recognized.

\section{REFERENCES}

[1] Žiljak, J., Jurecic, D., \& Žiljak, V. (2018). Packaging Design with Hidden near Infrared Colour Separation. Technical gazette, 25(3), 211-215. https://doi.org/10.17559/TV-20170705114921

[2] Rajković, I. \& Žiljak, V. (2016). Usage of ZRGB video camera as a detection and protection system and development of invisible infrared design. Polytechnic \& Design, 4(1), 54-59. https://doi.org/10.19279/TVZ.PD.2016-4-1-0777

[3]. Stanimirović Žiljak, I., Politis, A. E., Stanic Loknar, N., \& Janko K. (2014). The future of print, online and mobile media with use of individualized QR codes in V and NIR spectrum. $46^{\text {th }}$ Annual IC International Conference on Graphic Arts and Media Technology, Management and Education, Athens Greece, 71-78, ISBN 978-618-81734-0-8

[4] Žiljak, V., Žiljak Gršić, J., Jurecic, D., \& Jelicic, T. (2019). Near-infrared spectroscopy and hidden graphics applied in printing security documents in the offset technique. Tehnicki glasnik, 13(4), 311-314. https://doi.org/10.31803/tg-20191004140247

[5] Žiljak Gršić, J. (2017). Near infrared spectroscopy in print technology. Polytechnic \& Design, 5(1), 32-36. https://doi.org/10.19279/TVZ.PD.2017-5-1-05 https://doi.org/10.19279/TVZ.PD.2017-5-1-05-en

[6] Animation: https://jana.ziljak.hr/beef.mp4, https://jana.ziljak.hr/beef.swf

[7] Projectina Docucenter 4500, SP-2000 color spectroscopy module \& PAG B50 custom designed with 24 barrier filters; http://forensictechnology.com/projectina/
Authors' contacts:

Dražen Crčić

Modus Infinitum d.o.o.

49247 Zlatar Bistrica, Croatia

drazen.crcic@gmail.com

Jana Žiljak Gršić

Zagreb University of Applied Sciences,

Vrbik 8, 10000 Zagreb, Croatia

jana@ziljak.hr

Denis Jurečić

Faculty of Graphic Arts,

University of Zagreb,

Getaldićeva 2, 10000 Zagreb, Croatia

denis.jurecic@grf.hr 